\section{Mononuclear cells killing Cryptococcus}

Diamond in his report ${ }^{1}$ has presented by no means a new discovery; Aronson and Kletter ${ }^{2}$ have already demonstrated the in vitro killing of extracellular Cryptococcus neoformans by rabbit monocytes even in presence of normal rabbit serum. Monocytes do not necessarily require the presence of specific antibody to kill cryptococcus extracellularly but rather the presence of suitable opsonins which presumably enable the pseudopodia of the phagocytes to adhere firmly to the capsule of this fungus. Rabbit, guinea pig and human sera (but not mouse sera) seem to possess opsonins which are able to induce the phagocytosis of the cryptococci as well as the development of monocyte rings around them which, in turn, can destroy the enclosed parasites.

I feel that Diamond errs when he states that activated macrophages “... provided a preferentially favourable medium for intracellular growth of the fungus" and infers that host macrophages may be involved in the dissemination of the disease ${ }^{3}$. He drew these conclusions from experiments with human monocyte-derived macrophages activated in in vitro conditions with streptokinase-streptodornase and kept in culture for 3-7 d before inoculation of cryptococci. I have observed the extracellular and intracellular killing of cryptococci by murine macrophages (activated either nonspecifically or specifically) in certain conditions: that is, by in vivo-activated adherent cells kept in in vitro culture for only $2 \mathrm{~h}$ before the inoculation of cryptococci in the presence of normal rabbit serum $^{4,5}$; these sera lacked agglutinating capacity. Prolonged in vitro incubation of activated macrophages resulted in a corresponding decrease of their intracellular killing activity.

If phagocyte spreading of the infection in the intact host was involved, as suggested by Diamond, then the administration of drugs like cortisone which are known to promptly depress phagocyte numbers in general should localise this infection. On the contrary, however, it is known that cortisone treatment results in the dissemination of this disease $e^{6,7}$. Diamond's interpretation, that the extracellular killing of cryptococci in his system where $10 \mu \mathrm{g} \mathrm{ml}^{-1}$ of cytochalasin B was included to prevent phagocytosis of organisms, was due to antibodydependent killing by monocytes, may not be exactly correct.

Egg-white lysozyme and various cationic proteins from rabbit polymorphonucleocytes are fungicidal for C. neoformans ${ }^{8}$ and the addition of
2-10 $\mu \mathrm{g} \mathrm{ml}^{-1}$ of cytochalason B facilitates the release of lysosomal enzymes from macrophages by exocytosis ${ }^{8}$. Thus the extracellular killing of cryptococci by enzymes released from monocytes simply as a result of their incubation with cytochalasin $\mathbf{B}$ is a possibility which cannot be dismissed by the data Diamond presented.

\section{Institute for Medical}

K. K. SETHI

Microbiology and Immunology.

University of Bonn,

53 Bonn, West Germany

Diamond, R. D., Nature, 247, 148-149 (1974).

'Aronson, M., and Kletter, J., in Dynamic Aspects of Host-Parasite Relationships (edit. by Zuckerman A., and Weiss, D. W.), 1, 132 (Academic Press, New York and London, 1973).

${ }^{3}$ Diamond, R. G., and Bennett, J. E., Infect. Immun., 7, 231 (1973).

${ }^{4}$ Sethi, K. K., et al., in Abstr. Ann. Meet. Am. Soc. Microbiol., 131 (1973).

"Sethi, K. K., and Pelster, B., in Proc. Int. Workshop Macrophage Activation, Reisenberg, West Germany (Excerpta Medica, in the press).

${ }^{6}$ Sethi, K. K., Salfelder, K., and Schwarz, J., Mycopathologica et Mycologia Applicata, 27, 357 (1965).

Salfelder, K., in Handbuch der Speziellen Pathologischen Anatomie und Histologie (edit. by $\mathrm{E}$. Uehlinger), 3, 383 (Springer-Verlag. Berlin, 1970).

Gadebusch, H. H., and Johnson, A. G., J. infect. Dis., 116, 551 (1966).

${ }^{9}$ Davies, P., Allison, A. C., and Haswell, A. D., Biochem. J., 134, 33 (1973).

Dr Diamond REPLIES-Sethi seems to have overlooked or misinterpreted the major differences between my studies and those of Aronson and Kletter ${ }^{1}$. In contrast to their studies, the fungicidal mechanism which I described requires effector cells which are not necessarily monocytes, as well as specific antibody, but no heat-labile opsonins. The heat-labile opsonins which Aronson and Kletter referred to are almost certainly complement components, as detailed in studies of the role of the classical and alternate complement pathways in opsonisation of cryptococci and in the pathogenesis of cryptococcosis ${ }^{2-4}$

In my study of the possible role of fungicidal activity by non-phagocytic cells, mononuclear cell preparations had $20 \%$ or more monocytes mixed with lymphocytes. The effect of heat-labile opsonins was eliminated by heat and the role of other complement components was minimised by the large dilution $(1: 2,000$ or $1: 20,000)$ of antibody. No other serum was present. Unlike the studies of Aronson and Kletter, fungicidal activity occurred only in the presence of antibody, even when effector cells were obtained from four donors who had positive delayed skin tests to cryptococcal antigen.

Sethi's assumption that monocytes are responsible for the fungicidal activity is not warranted. In fact, I suggested an analogy to antibodydependent cell-mediated lysis of mammalian target cells, which is known to occur when all phagocytic cells have been eliminated from effector cell populations ${ }^{5}$. In subsequent studies, we used lymphoid cells depleted of phagocytic cells by incubation with carbonyl iron particles and passage through a magnetic field modified from the technique of Lundgren et al. ${ }^{6}$. Only $43.5 \%$ of a crytococcal inoculum survived after incubation for $4 \mathrm{~h}$. This fungicidal activity occurred only in the presence of antibody (R.D.D. and A. C. Allison, unpublished).

The role of the macrophage in immunity to cryptococcosis is another issue entirely, and one which is too complex to be thoroughly discussed here. In any case, corticosteroids have a wide range of effects, and I am surprised that Sethi assumes that depression of the number of phagocytes is the overriding corticosteroid effect on the immune response.

I did not study the mechanism of antibody-dependent killing and certainly did not assume that fungi were killed by monocytes, as Sethi states. Monocytes may well have this killing capacity, and lysosomal enzyme release may well be important. Preliminary studies with my coworkers (R.D.C., C. Cardella, P. Davies, and A. C. Allison, unpublished) have shown, however, that supernatants rich in lysosomal enzymes released from macrophages ${ }^{7}$ do not kill cryptococci and that cryptococci induce detectable specific lysosomal enzyme release only in the presence of heatlabile opsonins. Anti-cryptococcal antibody augments this release only slightly, if at all.

I regret that Sethi has misunderstood my work, but appreciate the opportunity to rectify the inadvertent omission of reference to the work of Aronson and Kletter, which was called to my attention afiter my manuscript appeared.

\section{Michael Reese Medical Center,}

\section{Chicago, Illinois 60616}

Aronson, M., and Kletter, J., in Dynamic Aspects of Host-Parasite Relationships (edit. by Zuckerman, A., and Weiss, D. W.), 1, 132 (Academic Press, New York and London, 1973)

Diamond, R. D., May, J. E., Kane, M. A., Frank, M. M., and Bennet, J. E., Clin. Res., 21, 597 (1973).

Diamond, R. D., May, J. E., Kane, M. A., Frank, M. M., and Bennett, J. E.,J. Immun., 112, 2260 (1974)

- Diamond, R. D. May, J. E Kane, M. A.. Frank, M. M.. and Bennett, J. E.. Proc. Soc exp. Biol. Med., 144, 312 (1973).

"Holm, G., and Perlmann, P. J., J. exp. Med., 125. 721 (1967).

6 Lundgren, G., Zukoski, C. H. F., and Möller, G., Clin. exp. Immun., 3, 817 (1968)

'Davies, P.. Allison, A. C., Ackerman, J., Butterfield, A.. and Williams, S., Nature, 251, 423 (1974). 